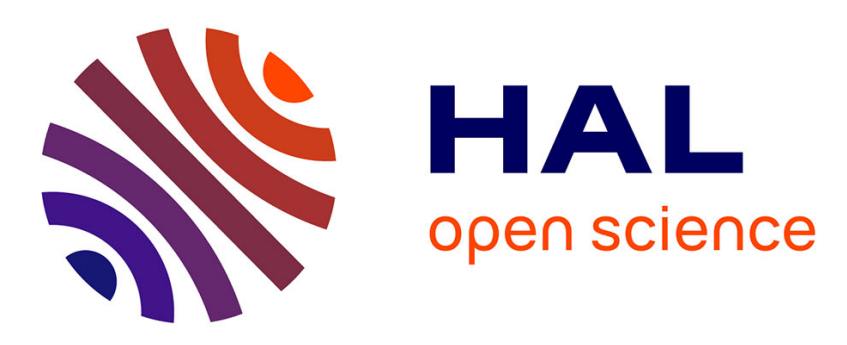

\title{
Ultrafast nonlinear phonon response of few-layer hexagonal boron nitride
}

\author{
Taehee Kang, Jia Zhang, Achintya Kundu, Klaus Reimann, Michael Woerner, \\ Thomas Elsaesser, Bernard Gil, Guillaume Cassabois, Christos Flytzanis, \\ Giorgia Fugallo, et al.
}

\section{To cite this version:}

Taehee Kang, Jia Zhang, Achintya Kundu, Klaus Reimann, Michael Woerner, et al.. Ultrafast nonlinear phonon response of few-layer hexagonal boron nitride. Physical Review B, 2021, 104 (14), pp.L140302. 10.1103/PhysRevB.104.L140302 . hal-03374670

\author{
HAL Id: hal-03374670 \\ https://hal.science/hal-03374670
}

Submitted on 12 Oct 2021

HAL is a multi-disciplinary open access archive for the deposit and dissemination of scientific research documents, whether they are published or not. The documents may come from teaching and research institutions in France or abroad, or from public or private research centers.
L'archive ouverte pluridisciplinaire HAL, est destinée au dépôt et à la diffusion de documents scientifiques de niveau recherche, publiés ou non, émanant des établissements d'enseignement et de recherche français ou étrangers, des laboratoires publics ou privés. 


\title{
Ultrafast nonlinear phonon response of few-layer hexagonal boron nitride
}

\author{
Taehee Kang, Jia Zhang, Achintya Kundu, Klaus Reimann, Michael Woerner, and Thomas Elsaesser*
}

Max-Born-Institut für Nichtlineare Optik und Kurzzeitspektroskopie, 12489 Berlin, Germany

Bernard Gil and Guillaume Cassabois

Laboratoire Charles Coulomb (L2C), UMR 5221 CNRS-Université de Montpellier, F-34095 Montpellier, France

Christos Flytzanis

Laboratoire de Physique, École Normale Supérieure, Université PSL, F-75231 Paris, France

Giorgia Fugallo

CNRS, UMR 6607, Laboratorie de Thermique et Energie de Nantes (LTeN) Polytech'Nantes, Université de Nantes, F-44306, Nantes, France

Michele Lazzeri

Sorbonne Université, CNRS UMR 7590, MNHN, IMPMC, F-75005 Paris, France

Ryan Page and Debdeep Jena

Cornell University, Department of Materials Science and Engineering, Ithaca, New York 148453, USA

(Dated: October 12, 2021)

\begin{abstract}
Nonequilibrium dynamics of TO phonons and low-frequency interlayer shear modes are mapped in femtosecond mid-infrared pump-probe experiments. Time-resolved changes of TO phonon absorption reveal a TO phonon lifetime of $1.2 \mathrm{ps}$, while the low-frequency excitations decay with a time constant of approximately 20 ps. The coupling of intralayer TO and interlayer motions manifests in a quasi-instantaneous redshift of the TO phonon resonance by some $10 \mathrm{~cm}^{-1}$. Theoretical calculations account for the coupling scenario and underline the relevance of inter-phonon interactions for the nonlinear phonon response of few-layer materials.
\end{abstract}

The crystal structure of hexagonal boron nitride $(\mathrm{hBN})$ consists of covalently bound 6-rings of boron and nitrogen atoms arranged in two-dimensional layers [1]. Adjacent layers are coupled via much weaker van der Waals interactions, making the material highly anisotropic. Mono- and few-layer $\mathrm{hBN}$ have received strong interest as a prototypical quasi-twodimensional quantum material [2], holding strong potential for synthesizing van der Waals heterostructures [3] and applications in optoelectronics [4], nanophotonics, and phonon polaritonics [5]. The electronic structure [6, 7], the linear and the nonlinear optical properties $[8,9]$ of few-layer hBN have been studied in some detail, whereas insight in carrier and phonon dynamics has remained very limited [10].

The anisotropic structure of $\mathrm{hBN}$ results in distinctly different frequencies of phonons connected with intra- and interplane atomic motions. Phonon modes of bulk hBN have been studied by infrared and Raman spectroscopy [11-15], inelastic x-ray scattering [13], and theoretical calculations $[16,17]$. At the $\Gamma$ point $(q=0)$, the infrared-active transverseoptical (TO) phonon of $E_{1 u}$ symmetry displays a frequency of $1367 \mathrm{~cm}^{-1}\left(E_{T O}=169.5 \mathrm{meV}\right)$, nearly identical to a Ramanactive phonon of $E_{2 g}$ symmetry. Both modes are connected with different in-plane atomic motions, as shown for the TO phonon in Fig. 1(a) (inset). Second-order Raman spectra suggest a TO phonon anharmonicity on the order of $5 \mathrm{~cm}^{-1}$ [13].

Lifetimes of the TO phonon of bulk hBN have been estimated from the damping term in numerical line shape fits of the reststrahlen band, giving values from 0.8 to $1.3 \mathrm{ps}$ for samples with the natural isotope variation of boron $[5,15,18]$.
An analysis of the Raman line shapes of the $E_{2 g}$ mode at $1367 \mathrm{~cm}^{-1}$ suggests a similar lifetime of 0.7 to $1 \mathrm{ps}$. For both modes, similar decay channels mediated via three- and four-phonon anharmonic couplings have been proposed [18].

Motions of the stacked $\mathrm{BN}$ planes relative to each other give rise to rigid-plane shear and compression modes at low energy. In bulk hBN, the Raman-active horizontally-polarized rigid-plane shear mode arises at $54 \mathrm{~cm}^{-1}\left(E_{S H}=6.74 \mathrm{meV}\right)$. In hBN with a finite number $N$ of BN layers, the discretization of the $k$-vector perpendicular to the layers results in an increasing number of optical phonon branches. Theory $[16,17]$ predicts a splitting of the bulk rigid-plane modes in $N-1$ compression modes and $N-1$ doubly degenerate shear modes, the latter at energies below $E_{S H}$. So far, experimental results on such modes are very limited [19] and couplings of intra- and interlayer modes unknown.

For a basic physical understanding of the low-frequency dielectric properties of $\mathrm{hBN}$ and applications in phonon polaritonics, insight in phonon-phonon interactions and the resulting nonequilibrium dynamics is mandatory. Moreover, coupled phonon excitations are relevant for energy relaxation and heat transport, including the interplay of in-plane and interplane motions. In this Letter, we report the first study of the nonlinear phonon response of few-layer hBN. Upon femtosecond mid-infrared excitation via the TO phonon absorption band, the resulting ultrafast dynamics exhibit both a pronounced coupling of inter-layer and TO phonon modes and population relaxation processes of the coupled modes on different time scales. The anharmonic phonon-phonon coupling 
is accounted for by in-depth theoretical calculations.

The few-layer hBN sample studied here was grown directly on a sapphire substrate and has an average thickness of $2.5 \mathrm{~nm}$, corresponding to $N=8$ to $9 \mathrm{hBN}$ layers. For such $N$, the rigid-plane shear phonons are expected at frequencies below the bulk shear mode at $54 \mathrm{~cm}^{-1}[17,19]$. A temporally and spectrally resolved pump-probe approach with femtosecond mid-infrared pulses is employed to map nonequilibrium phonon dynamics. As depicted schematically in Fig. 1(b) (inset), the $s$-polarized (TE) pump and probe pulses were reflected from a gold-coated prism and hit the sample under slightly different angles of incidence. The probe beam reflected from the sample passed the second facet of the prism, was spectrally dispersed in a monochromator, and detected with a 64-element $\mathrm{HgCdTe}$ detector array (spectral resolution $2 \mathrm{~cm}^{-1}$ ). A reference pulse reflected from an unexcited part of the sample was detected by a second array. Details of sample preparation, interaction geometry and mid-infrared pulse generation are presented in the supplemental material (SM).

The linear reflectivity spectrum [Fig. 1(a)] of the hBN sample exhibits the TO phonon resonance at $1366 \mathrm{~cm}^{-1}$, in agreement with an attenuated total reflection spectrum of the sample (Fig. S2 of the SM). The width of the resonance has a value $\Delta v_{T O} \approx 15 \mathrm{~cm}^{-1}$, which is about twice as large as values derived from the reststrahlen band of high-quality bulk hBN. We assign this discrepancy to structural imperfections of the few-layer sample. A more detailed analysis of the reflectivity spectrum is presented in the SM.

In Figs. 1(b,c), pump-probe spectra measured with pump energies $E_{p}=70 \mathrm{~nJ}$ and $E_{p}=1.0 \mu \mathrm{J}$ are summarized (symbols). The change of reflectivity $\Delta R / R_{0}$ is plotted as a function of probe frequency $v_{p r}$ for the delay times given in panel (c). From the pump fluence and the TO phonon absorption of the sample, one estimates an excitation density of $6.5 \times 10^{19} \mathrm{~cm}^{-3}$ for $\mathrm{E}_{p}=70 \mathrm{~nJ}$ and $9.5 \times 10^{20} \mathrm{~cm}^{-3}$ for $\mathrm{E}_{p}=1.0 \mu \mathrm{J}$. Both numbers are much smaller than the TO phonon density of states of approximately $5 \times 10^{22} \mathrm{~cm}^{-3}$, as estimated from an Einstein model. Thus, multiple excitations to higher phonon states are negligible.

All transient spectra display a decrease of reflectivity at high probe frequencies, corresponding to an absorption decrease, and an increase of reflectivity/absorption at low probe frequencies. The spectra for $E_{p}=70 \mathrm{~nJ}$ [Fig. 1(b)] are of a dispersive shape, which is similar for all time delays and within the TO phonon line observed in linear reflectivity [thick solid line in panel (b)]. In contrast, the spectral envelope of the positive and negative $\Delta R$ and the position of the zero crossing change with delay time for the higher $E_{p}=1 \mu \mathrm{J}$ [Fig. 1(c)].

The time resolved pump-probe traces shown in Fig. 2 were recorded at the probe frequencies marked by arrows in Fig. 1(c). Around delay zero, the transients show a fast modulation originating from nonlinear cross-phase modulation of pump and probe pulses. All transients measured with $E_{p}=70$ nJ [Fig. 2(a)] exhibit a biphasic decay with relative amplitudes depending on probe frequency. Such kinetics are reproduced by fitting two instantly rising contributions with

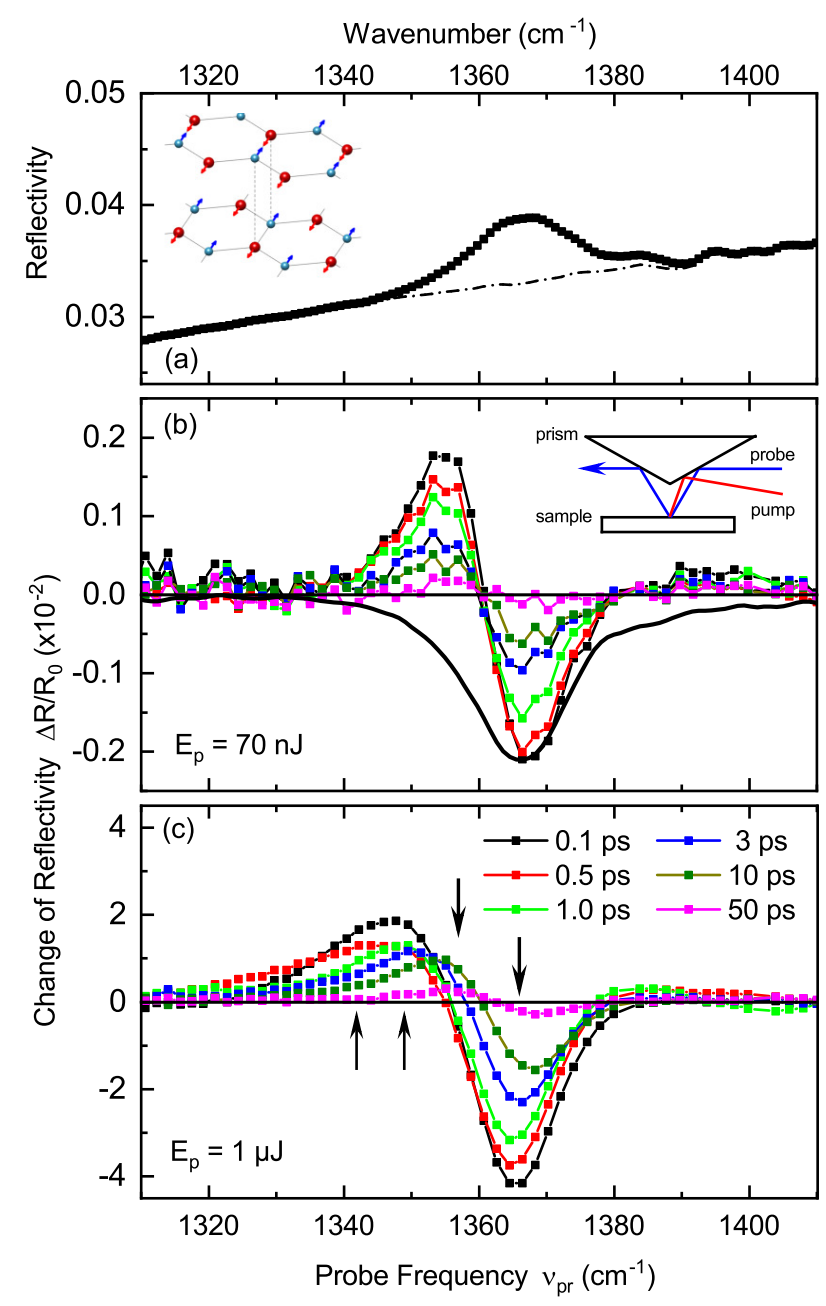

FIG. 1. (a) Reflecticity spectrum of the few-layer hBN sample in the range of the TO phonon resonance at $1366 \mathrm{~cm}^{-1}$ (symbols). Dash-dotted line: experimental reflectivity spectrum of the sapphire substrate. Inset: Atomic elongations connected with the TO phonon of hBN. (b) Transient reflectivity spectra (symbols) measured with a pump energy $E_{p}=70 \mathrm{~nJ}$. The change of reflectivity $\Delta R / R_{0}=\left(R-R_{0}\right) / R_{0}$ is plotted as a function of probe frequency for different delay times $\left(R, R_{0}\right.$ : reflectivity of the sample with and without excitation). Solid lines are to guide the eye. The thick solid line gives the inverted and scaled reflectivity spectrum from panel (a) after subtraction of the substrate reflectivity. Inset: Pump-probe geometry applied in the experiments. (c) Same as (b) for a pump energy $E_{p}=1.0 \mu \mathrm{J}$. The arrows mark the probe frequencies at which the transients in Fig. 2 were recorded.

decay times of $1.2 \pm 0.3$ ps and $22 \pm 5$ ps to the data points [solid lines in Figs. 2(a), (b)]. The long decay time was validated by data extending to delay times of 60 ps (see SM). The transients recorded with $E_{p}=1 \mu \mathrm{J}$ reveal a more complex kinetic behavior. The decays observed at $v_{p r}=1342$ and $1366 \mathrm{~cm}^{-1}$ follow biexponential decays with time constants close to the data for $E_{p}=70 \mathrm{~nJ}$. In contrast, the reflectivity changes at $v_{p r}=1349$ and $1357 \mathrm{~cm}^{-1}$ are not accounted for by rate-like kinetics. This fact reflects the reshaping of the 


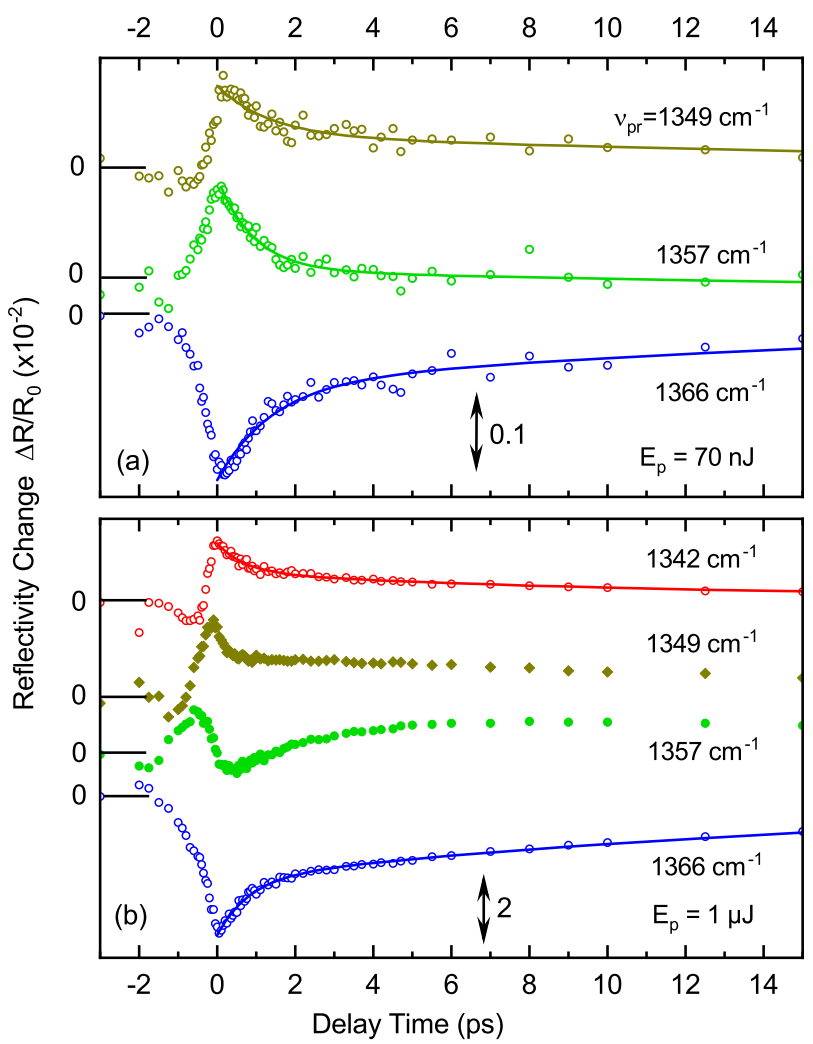

FIG. 2. (a) Time resolved pump-probe traces recorded at fixed probe frequencies $v_{p r}$ [cf. arrows in Fig. 1(c)] with a pump energy $E_{p}=$ $70 \mathrm{~nJ}$ (symbols). The change of reflectivity $\Delta R / R_{0}$ is plotted as a function of pump-probe delay. The solid lines are numerical fits to the data and represent biexponential decays with time constants of $1.2 \mathrm{ps}$ and $22 \mathrm{ps}$. (b) Same for $E_{p}=1.0 \mu \mathrm{J}$.

pump-probe spectra [cf. Fig. 1(c)] and the frequency shift of the zero crossing with delay time.

Experiments with pump pulses of a reduced bandwidth $\Delta v_{p}$ give additional insight in the nonlinear phonon response. The pump energy $E_{p}$ was adjusted to implement a similar excitation level for $\Delta v_{p}=20,32,54$, and $200 \mathrm{~cm}^{-1}$ (FWHM). Pump-probe spectra for a delay time $t_{D}=3 \mathrm{ps}$ after the fast signal decay [Fig. 3(a)] display identical envelopes for the different $\Delta v_{p}$. The time evolution of reflectivity changes at a fixed probe frequency (not shown) is independent from $\Delta v_{p}$ as well. In Fig. 3(b), the frequency positions of the positive and negative peaks in the spectra for $t_{D}=3 \mathrm{ps}$ are plotted vs $\Delta v_{p}$. Figure 3(c) shows the frequency position of the positive peak (solid symbols) and the first moment $M=\left(\int v g(v) d v\right) / \int g(v) d v$ of the redshifted positive component $g(v)$ of the pump-probe spectra as a function of $E_{p}$ (open symbols). Both quantities exhibit a redshift increasing roughly linearly with $E_{p}$.

We now discuss the mechanisms behind the ultrafast nonlinear phonon response with the support of first principles evaluations [23-25] of phonon lifetimes, anharmonic frequency shifts and inter-phonon anharmonic couplings [26, 32,

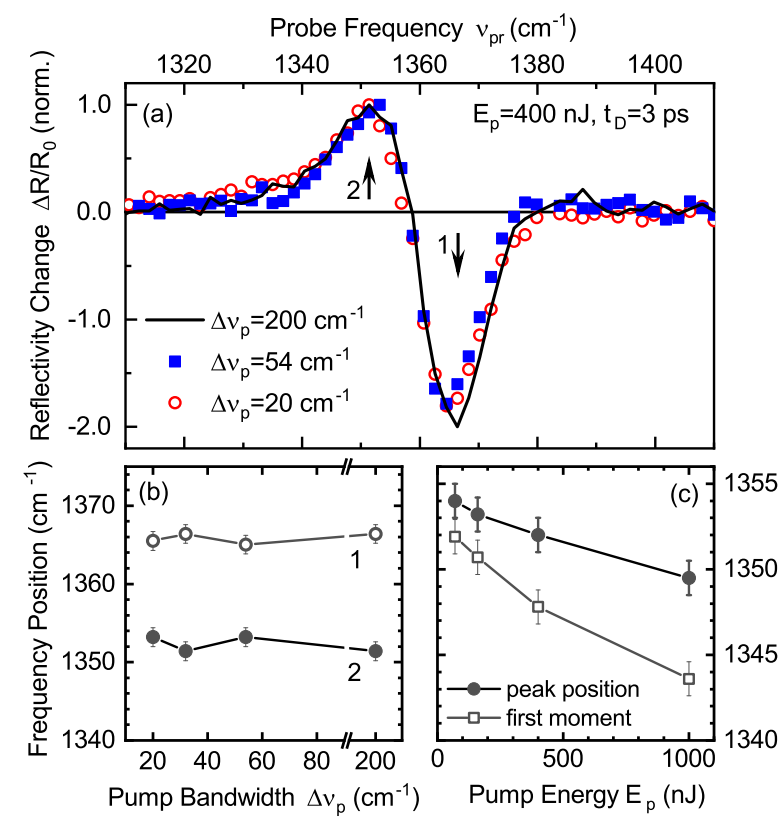

(d)

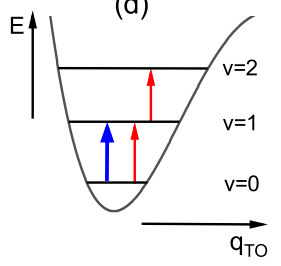

(e)

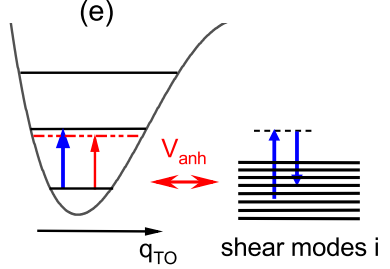

FIG. 3. Pump-probe spectra at a delay $t_{D}=3$ ps recorded with pump bandwidths $\Delta v_{p}$. The spectra are normalized to the maximum positive $\Delta R / R_{0}$. The pump energy was $E_{p}=400 \mathrm{~nJ}$ for $\Delta v_{p}=200 \mathrm{~cm}^{-1}$. For a reduced $\Delta v_{p}, E_{p}$ was adjusted to make the amount of absorbed pump energy identical to the data for $E_{p}=400 \mathrm{~nJ}$. (b) Frequency positions of the maximum negative (1) and positive (2) reflectivity changes as a function of $\Delta v_{p}$. (c) Peak position and first moment of the positive reflectivity change as a function of $E_{p}$ for excitation with $\Delta v_{p}=200 \mathrm{~cm}^{-1}$ (d) Schematic of the anharmonic TO phonon potential along the coordinate $q_{T O}$ with optical transitions relevant for excitation (blue arrow) and probing (red arrows). (e) Anharmonic TO phonon potential along $q_{T O}$ and vibrational levels of low-frequency shear modes $i$ with a Raman excitation process (blue arrows). The TO phonon and shear modes are anharmonically coupled via $\mathrm{V}_{\text {anh }}$.

33]. The theoretical methods are accounted for in the SM. We recall that the TO phonon potential energy surface shows a small anharmonicity along the $q_{T O}$ coordinate of $5 \mathrm{~cm}^{-1}$ [13]. As schematically shown in Fig. 3(d), this anharmonicity leads to a redshift of the $v=1$ to 2 compared to the fundamental $v=0$ to 1 transition. Resonant excitation by the pump pulses [blue arrow in Fig. 3(d)] results in a population decrease in the $v=0$ and an increase in the $v=1$ TO phonon state. Concomitantly, the absorption/reflectivity on the $v=0$ to 1 transition decreases, due to depopulation of the $v=0$ state and stimulated emission from the $v=1$ state. In parallel, a redshifted absorption/reflectivity increase arises on the $v=1$ to 2 transition. The probe pulse [red arrows in Fig. 3(d)] maps such 
changes.

The dispersive shape of the transient spectra in Figs. 1(b,c) is in line with this picture. For a pump energy $E_{p}=70 \mathrm{~nJ}$, all reflectivity changes occur within the steady-state TO phonon peak [thick solid line in Fig. 1(b)], reflecting the small diagonal anharmonicity. The time resolved transients in Fig. 2(a) display a first decay time of $1.2 \mathrm{ps,} \mathrm{which} \mathrm{we} \mathrm{assign} \mathrm{to}$ the population decay of the $v=1$ state of the TO phonon. The 1.2-ps decay measured here directly supports indirect lifetime estimates from vibrational linewidths [5, 18]. For $E_{p}=1 \mu \mathrm{J}$, one observes a similar 1.2-ps decay at $v_{p r}=1342$ and $1366 \mathrm{~cm}^{-1}$.

Relaxation of the $v=1 \mathrm{TO}$ phonon population is connected with a population transfer to anharmonically coupled phonons at lower frequency $[14,15,18]$. The theoretical calculations of Ref. 18 suggest a predominant 4-phonon decay channel, invoking the zone-center $A_{2 u}$ phonon at $820 \mathrm{~cm}^{-1}$ and ZO/ZA phonons at the $\mathrm{M}, \mathrm{K}$ points of the Brillouin zone with frequencies around $300 \mathrm{~cm}^{-1}$. A relaxation scheme and more detailed discussion based on our calculations is presented in the SM. The calculated TO-phonon lifetimes of 1 ps [18] and 1.78 ps (SM) are close to the experimental value of $1.2 \mathrm{ps}$.

The pump-probe spectra (Fig. 1) display a redshift of the fundamental ( $v=0$ to 1 ) TO phonon absorption even after the decay of the TO $v=1$ population, i.e., with the TO phonons in their $v=0$ state. The non-exponential time-resolved transients measured with $E_{p}=1 \mu \mathrm{J}$ at $v_{p r}=1349$ and $1357 \mathrm{~cm}^{-1}$ [Fig. 2(b)] demonstrate the onset of this redshift at early delays during the decay of the $v=1$ state, and, together with all other transients in Fig. 2, their decay with a time constant of 22 ps. The redshift of the TO phonon transition is due to the intermode anharmonic coupling to a subset of low-frequency phonons excited by the pump pulse. As sketched in Fig. 3(e), a Raman process which is resonantly enhanced by the TO transition dipole, excites low-frequency Raman active phonons $q_{S H, i}$. This excitation mechanism is nonselective and generates excess populations of all Raman-active modes within the pump bandwidth. The nonequilibrium populations of lowfrequency modes redshift the TO phonon transition via the anharmonic coupling $V_{a n h}$. The redshift at a delay $t_{D}=3 \mathrm{ps}$ after the TO phonon decay scales with the pump energy $E_{p}$ [Fig. 3(c)], i.e., the generated excess population, and decays with a time constant of 22 ps by population relaxation of the low-frequency phonon states.

The experiments with reduced pump bandwidth $\Delta v_{p}$ (Fig. 3) show that the redshift at $t_{D}=3$ ps scales with $E_{p}$ but not with $\Delta v_{p}$. The data for $\Delta v_{p}=20 \mathrm{~cm}^{-1}$ (FWHM), however, set an upper limit of $30-40 \mathrm{~cm}^{-1}$ for the frequency of the phonons inducing the redshift. While optically accessible phonons below $50 \mathrm{~cm}^{-1}$ are absent in bulk hBN, a subset of Raman active interlayer shear modes $i$ and 1-2 compression modes of few-layer hBN shows sub-40 $\mathrm{cm}^{-1}$ frequencies [cf. SM, [17]]. The observed TO redshift is assigned to a coupling with such low-frequency phonons in which nonequilibrium populations are generated by the Raman pump mechanism. Independent evidence for the exis- tence of low-frequency shear and compression modes comes from the Raman spectra of Ref. 19, displaying a broad lowfrequency phonon signal superimposed by a sharp line due to the strongest Raman line at the highest shear-mode frequency [34].

This picture relies on the concept that due to optical selection rule the pump pulse excites only phonons with zero wavevector, i.e., in an infinitesimally small volume in the Brillouin zone (BZ). If we indicate the phonon population as $n(\mathbf{q})$, where $\mathbf{q}$ is the wavevector, immediately after the pump excitation we have $n(\mathbf{q})=n_{0}(\mathbf{q})+A \delta(\mathbf{q})$ where $n_{0}(\mathbf{Q})$ is the thermal $(T=300 \mathrm{~K})$ population and $\delta$ is the Dirac distribution [35]. Such a distribution does not alter the phonon lifetimes, which are determined by the population distribution over all the BZ (and not by what happens only at $\mathbf{q}=0$ ). However, it can induce a frequency shift of the TO mode through anharmonic coupling with the low-energy shear modes at the $\mathrm{BZ}$ center. Interestingly, in bulk hBN the TO and the lowenergy shear modes are weakly coupled, with a predominant contribution of 4-phonon processes and a coupling term of the order of $-0.02 \mathrm{~cm}^{-1}$. With this weak coupling in order to experimentally observe a TO shift $\Delta v_{T O}=10 \mathrm{~cm}^{-1}$, we should assume $A \approx \Delta v_{T O} /\left(0.02 \mathrm{~cm}^{-1}\right) \sim 500$. For the bulk $E_{S H}=6.7 \mathrm{meV}$, this value of $A$ corresponds to an effective energy per unit cell of $\sim 1 \mathrm{eV}$ to be provided by the pump pulse, a value prohibitively high under the present experimental conditions.

On the contrary, the generation of a TO shift $\Delta \nu_{T O}=10$ $\mathrm{cm}^{-1}$ becomes possible when passing from bulk to few-layer $\mathrm{hBN}$. Our calculations show that the coupling term displays a strong dispersion along the $\mathrm{z}$-axis and increases up to two orders of magnitude. For a maximum shear-mode frequency of $30 \mathrm{~cm}^{-1}$ (cf. Fig. 3), such strong coupling shrinks the excitation energy required per unit cell to roughly $20 \mathrm{meV}$, well in the range of the experiment. Our picture is further supported by the calculated lifetime of the low-energy shear modes of $\tau_{S H}=25.81 \mathrm{ps}$, close to the experimental decay time of $22 \mathrm{ps}$. The large difference between $\tau_{S H}$ and the 1.2-ps TO phonon lifetime assures, as experimentally observed, that the TO nonlinear signal decreases while the redshift remains the same during the TO decay time.

In conclusion, the ultrafast nonlinear phonon response of few-layer hexagonal boron nitride reveals a TO phonon lifetime of $1.2 \mathrm{ps}$ and a 22-ps decay time of low-frequency phonons. Nonequilibrium populations of rigid-plane shear modes below $30 \mathrm{~cm}^{-1}$ induce a transient redshift of the TO phonon resonance, a direct manifestation of the anharmonic coupling between the TO-intralayer and interlayer degrees of freedom. This coupling is highly relevant for future extensions of phonon polaritonics into the nonlinear regime.

\footnotetext{
* elsasser@mbi-berlin.de

[1] R. S. Pease, Crystal structure of boron nitride, Nature 165, 722
} 
(1950).

[2] Y. Shi, C. Hamsen, X. Jia, K. Kang Kim, A. Reina, M. Hofmann, A. Long Hsu, K. Zhang, H. Li, Z.-Y. Juang, M. S. Dresselhaus, L.-J. Li, and J. Kong, Synthesis of few-layer hexagonal boron nitride thin film by chemical vapor deposition, Nanolett. 10, 4134 (2010).

[3] A. K. Geim and I. V. Grigorieva, Van der Waals heterostructures, Nature 499, 419 (2013)

[4] J. D. Caldwell, I. Aharonovich, G. Cassabois, J. H. Edgar, B. Gil, and D. N. Basov, Photonics with hexagonal boron nitride, Nature Rev. Mat. 4, 552 (2019)

[5] A. J. Giles et al., Ultralow-loss polaritons in isotopically pure boron nitride, Nature Mat. 17, 134 (2018).

[6] G. Cassabois, P. Valvin, and B. Gil, Hexagonal boron nitride is an indirect bandgap semiconductor, Nature Photon. 10, 262 (2016).

[7] L. Schué, L. Sponza, A. Plaud, H. Bensalah, K. Watanabe, T. Taniguchi, F. Ducastelle, A. Loiseau, and J. Barjon, Bright luminescence from indirect and strongly bound excitons in h-BN, Phys. Rev. Lett, 122, 067401 (2019).

[8] Y. Li, Y. Rao, K. F. Mak, Y. You, S. Wang, C. R. Dean, and T. F. Heinz, Probing symmetry properties of few-layer $\mathrm{MoS}_{2}$ and hBN by optical second-harmonic generation, Nanolett. 13, 3329 (2013).

[9] C.-J. Kim, L. Brown, M. W. Graham, R. Hovden, R. W. Havener, P. L. McEuen, D. A. Muller, and J. Park, Stacking order dependent second harmonic generation and topological defects in h-BN bilayers, Nanolett. 13, 5660 (2013).

[10] D. Golla, A. Brasington, B. J. LeRoy, and A. Sandhu, Ultrafast relaxation of hot phonons in graphene-hBN heterostructures, APL Mat. 5, 056101 (2017).

[11] R. Geick, C. H. Perry, and G. Rupprecht, Normal modes in hexagonal boron nitride, Phys. Rev. 146, 543 (1966).

[12] S. Reich, A. C. Ferrari, R. Arenal, A. Loiseau, I. Bello, and J. Robertson, Resonant Raman scattering in cubic and hexagonal boron nitride, Phys. Rev. B 71, 205201 (2005).

[13] J. Serrano, A. Bosak, R. Arenal, M. Krisch, K. Watanabe, T. Taniguchi, H. Kanda, A. Rubio, and L. Wirtz, Vibrational properties of hexagonal boron nitride: Inelastic $\mathrm{x}$-ray scattering and ab-initio calculations, Phys. Rev. Lett. 98, 095503 (2007).

[14] R. Cuscó, B. Gil, G. Cassabois, and L. Artús, Temperature dependence of Raman-active phonons and anharmonic interactions in layered hexagonal BN, Phys. Rev. B 94, 155435 (2016).

[15] R. Cuscó, L. Artús, J. H. Edgar, S. Liu, G. Cassabois, and B. Gil, Isotope effects on phonon anharmonicity in layered van der Waals crystals: Isotopically pure hexagonal boron nitride, Phys. Rev. B 97, 155435 (2018).

[16] K. H. Michel and B. Verberck, Phonon dispersions and piezoelectricity in bulk and multilayers of hexagonal boron nitride, Phys. Rev. B 83, 115328 (2011).

[17] K. H. Michel and B. Verberck, Theory of rigid-plane phonon modes in layered crystals, Phys. Rev. B 85, 094303 (2012).

[18] A. Segura, R. Cuscó, T. Taniguchi, K. Watanabe, and L. Artús, Long lifetime of the $\mathrm{E}_{1 u}$ in-plane infrared-active modes of hBN, Phys. Rev. B 101, 235203 (2020).

[19] I. Stenger, L. Schué, M. Boukhicha, B. Berini, B. Plaçais, L. Loiseau, and J. Barjon, Low frequency Raman spectroscopy of few-atomic-layer thick hBN crystals. 2D Mater. 4, 031003 (2017).

[20] N. E. Levinger, R. Costard, E. T. J. Nibbering, and T. Elsaesser, Ultrafast energy migration pathways in self-assembled phos- pholipids interacting with confined water, J. Phys. Chem. A 115, 11952 (2011).

[21] R. Page, J. Casamento, Y. Cho, S. Rouvimov, H.G. Xing, and D. Jena, Rotationally aligned hexagonal boron nitride on sapphire by high-temperature molecular beam epitaxy, Phys. Rev. Mater. 3, 064001 (2019).

[22] D. W. Berreman, Infrared absorption at longitudinal optical frequency in cubic crystal films, Phys. Rev. 130, 2193 (1963).

[23] P. Hohenberg and W. Kohn, Inhomogeneous electron gas, Phys. Rev. 136B, 864 (1964)

[24] W. Kohn and L. J. Sham, Self-consistent equations including exchange and correlation effects, Phys. Rev. 140A, 1133 (1965).

[25] S. Baroni, S. de Gironcoli, A. Dal Corso, and P. Giannozzi, Phonons and related crystal properties from density-functional perturbation theory, Rev. Mod. Phys. 73, 515 (2001).

[26] DFT calculations were done with the quantum espresso package [27] within pseudopotential [28] and the local density approximation [29] (110 Ry plane-waves cut-off and $12 \times 12 \times 8$ shifted electronic integration grid). Phonon frequencies were obtained on a mesh $8 \times 8 \times 6$ grid $\mathrm{f} \mathrm{k}$ points and interpolated to arbitrary wave vectors for the calculation of phonon dispersion and phonon density of states. All the scattering were first calculated on a $4 \times 4 \times 2$ phonon wavevectors grid and then Fourier interpolated on a $100 \times 100 \times 100$ grid (the procedure is described in detail in Refs. 30 and 31).

[27] P. Giannozzi, O. Andreussi, T. Brumme, O. Bunau, M. Buongiorno Nardelli, M. Calandra, R. Car, C. Cavazzoni, D. Ceresoli, M. Cococcioni, N. Colonna, I. Carnimeo, A. Dal Corso, S. de Gironcoli, P. Delugas, R. DiStasio, A. Ferretti, A. Floris, G. Fratesi, G. Fugallo, R. Gebauer, U. Gerstmann, F. Giustino, T. Gorni, J. Jia, M. Kawamura, H.Y. Ko, A. Kokalj, E. Kücükbenli, M. Lazzeri, M. Marsili, N. Marzari, F. Mauri, N.L. Nguyen, H.V. Nguyen, A. Otero-de-la-Roza, L. Paulatto, S. Poncé, D. Rocca, R. Sabatini, B. Santra, M. Schlipf, A.P. Seitsonen, S. Smogunov, I. Timrov, T. Thonhauser, P. Umari, N. Vast, and S. Baroni, Advanced capabilities for materials modelling with Quantum ESPRESSO, J. Phys.: Condens. Matter 29, 465901 (2017).

[28] N. Troullier and J. L. Martins, Efficient pseudopotentials for plane-wave calculations, Phys. Rev. B 43, 1993 (1991).

[29] J. P. Perdew and A. Zunger, Self-interaction correction to density-functional approximations for many-electron systems, Phys. Rev. B 23, 5048 (1981).

[30] M. Lazzeri and S. de Gironcoli, First-principles study of the thermal expansion of $\mathrm{Be}(10 \overline{1} 0)$, Phys. Rev. B 65, 245402 (2002).

[31] L. Paulatto, F. Mauri, and M. Lazzeri, Anharmonic properties from a generalized third-order $a b$ initio approach: Theory and applications to graphite and graphene, Phys. Rev. B 87, 214303 (2013).

[32] M. Lazzeri, M. Calandra, and F. Mauri, Anharmonic phonon frequency shift in $\mathrm{MgB}_{2}$, Phys. Rev. B 68, 220509(R) (2003).

[33] G. Fugallo, B. Rousseau, and M. Lazzeri, Infrared reflectance, transmittance, and emittance spectra of $\mathrm{MgO}$ from first principles, Phys. Rev. B 98, 184307 (2018).

[34] L. Liang, A. A. Puretzky, B. G. Sumpter, and V. Meunier, Interlayer bond polarizability model for stacking-dependent lowfrequency Raman scattering in layered materials, Nanoscale 9, 15340 (2017).

[35] $A$ is basically a phonon population resulting from a BoseEinstein statistics for a $50 \mathrm{~cm}^{-1}$ phonon. 